\title{
The Quest for a Fundamental Algorithm in X-Ray Fluorescence Analysis and Calibration
}

\author{
R.M. Rousseau* \\ Geological Survey of Canada, 601 Booth St., Room 707, Ottawa, Ontario, K1A 0E8, Canada
}

\begin{abstract}
The Fundamental Algorithm between concentration $\left(\mathrm{C}_{\mathrm{i}}\right)$ and intensity $\left(\mathrm{R}_{\mathrm{i}}\right)$ in X-ray fluorescence analysis is deduced from Sherman's equation without any approximation. Its explicit expression is thus theoretically exact and it corrects completely for all matrix effects on the intensity $R_{i}$ emitted by the analyte $i$ in a given specimen. This powerful algorithm combines the practical flexibility of the influence coefficient concept and the theoretical exactness of the Fundamental-Parameters method. In association with its innovative calibration procedure, the Fundamental Algorithm can be applied to the analysis of any sample type and offers maximum accuracy, limited only by the quality of sample preparation and the standards used.
\end{abstract}

\section{INTRODUCTION}

In the early seventies, $\mathrm{X}$-ray fluorescence (XRF) analysts were confronted with the problem to choose the most appropriate method for calculating the composition of the samples to be analyzed. In order to choose the appropriate method for a given analytical context, the author made an exhaustive study of the most popular methods published between 1954 and 1984. From this study, he learnt which methods are most performing, in which experimental conditions they must be applied, which features contribute to improve the quality of results and how to apply all this knowledge in practice. He attempted to regroup all these performing features in a new fundamental method valid for the complete analytical range $(0-100 \%)$. The resulting method was named the Fundamental Algorithm [1].

The Fundamental Algorithm between $C_{i}$ and $R_{i}$ in XRF analysis is deduced from Sherman's equation without any approximation. Its explicit expression is thus theoretically exact and it corrects completely for all matrix effects on the intensity $R_{i}$ emitted by the analyte $i$ in a given specimen. This powerful algorithm combines the practical flexibility of the influence coefficient concept and the theoretical exactness of the Fundamental-Parameters method. In association with its innovative calibration procedure, the Fundamental Algorithm can be applied to the analysis of any sample type and offers maximum accuracy, limited only by the quality of sample preparation and the standards used.

When a theoretical analytical method is implied, a special calibration procedure $[2,3]$ must be used, because it is well known that theory cannot account for the variations of all instrumental parameters. An innovative calibration procedure, comparing measured intensities to calculated ones, is then proposed.

Using the Fundamental Algorithm as basis, three optimized methods that cover the complete analytical range

*Address correspondence to this author at the Geological Survey of Canada, 601 Booth St., Room 707, Ottawa, Ontario, K1A 0E8, Canada;

E-mail: rroussea@nrcan.gc.ca
$(0-100 \%)$ are proposed. To learn how to apply these three optimized methods in practice, an experimental verification comparing given concentrations for $\mathrm{Fe}$ in a set of $\mathrm{Cr}-\mathrm{Fe}-\mathrm{Ni}$ specimens to the values calculated by the three different methods [4] is given.

\section{ORIGIN OF THE FUNDAMENTAL ALGORITHM}

The Fundamental Algorithm (FA) is the synthesis of 30 years of research and development on the correction for matrix effects in XRF analysis. A list of XRF scientists and a brief description of their main contributions to the FA, together with the publication year, is presented below. The ideas mentioned have been incorporated in the FA in one way or another, and contributed to make the FA one of the most efficient methods of correcting for matrix effects. They are:

\section{Sherman's equation [5]}

1966 Lachance and Traill [6] (LT):

Concept of the influence coefficient

Theoretical, binary, absorption

influence coefficients (alphas) for a

monochromatic excitation source

During the concentration calculation, there is no need for normalization to $100 \%$

1967 Claisse and Quintin [7] (CQ):

Generalization of the LT method for

matrix effect corrections

Introduce the weighting factor for each

incident wavelength $\lambda_{\mathrm{k}}$

Influence coefficients vary with

concentrations

1968 Gilfrich and Birks [8]: the first measured

incident spectrum

Criss and Birks [9]: the Fundamental-

Parameters method

Show how to use Sherman's equation

in practice

1970 Rousseau's Master thesis [10]:

For the first time, proposes the FA for

a monochromatic excitation source 
1973 de Jongh [11]:

Theoretical multi-element influence

coefficients can be calculated from the

Sherman equation

Calibration lines of measured intensities

versus calculated intensities

(or apparent concentration)

1974 Rasberry and Heinrich [12]:

Absorption and enhancement effects obey

to different mathematical rules and they

must be treated separately

1974 Rousseau and Claisse [13]:

Propose a new method to calculate

theoretical binary influence coefficients

in the CQ algorithm

1976 Tertian [14]:

Introduces the $\mathrm{C}_{\mathrm{M}}$ factor in the

CQ algorithm

1980 Criss [15]:

Proposes calculation of an initial

estimate of the sample composition

for calculating the $\alpha_{\mathrm{ij}}$ and $\varepsilon_{\mathrm{ij}}$ coefficients

once

1982 Rousseau:

First public presentation of the FA at the $31^{\text {st }}$ Annual Denver X-Ray Conference

1984 Rousseau [16]:

First publication on the Fundamental

Algorithm

1985 Pella, Feng and Small [17]:

Calculate intensity for each wavelength

of the incident spectrum

The unification of all these XRF methods has led to a new fundamental method called the Fundamental Algorithm. As it stands, the Fundamental Algorithm contains all that is needed to calculate a sample composition from measured Xray intensities and also provides a complete and accurate correction for all matrix effects. This relation is theoretically exact since it has been deduced from the Sherman equation without any approximation during the process of derivation.

In X-ray fluorescence analysis, the Sherman equation describes very well the relationship between the measured intensities emitted by a specimen and its composition. Indeed, in 1955, the physicist Jacob Sherman [5], from the Philadelphia Naval Shipyard (USA), published a detailed demonstration of an equation enabling one to calculate theoretical net X-ray intensities emitted by each element from a specimen of known composition when a polychromatic $\mathrm{X}$ ray beam irradiates it. This equation can be written in the form

$I_{i}=f\left(C_{i}, C_{j}, C_{k}, \ldots, C_{N}\right)$

where $\mathrm{I}_{\mathrm{X}}$ and $\mathrm{C}_{\mathrm{X}}$ are the net intensity and the concentration of the $\mathrm{N}$ elements present in the sample, respectively. Unfortunately, this equation is not reversible for transformation to

$\mathrm{C}_{\mathrm{i}}=\mathrm{f}\left(\mathrm{I}_{\mathrm{i}}, \mathrm{I}_{\mathrm{j}}, \mathrm{I}_{\mathrm{k}}, \ldots, \mathrm{I}_{\mathrm{N}}\right)$

which is required for the calculation of unknown sample compositions. In the initial Sherman equation, a factor of $1 / 2$ in the part of the secondary fluorescent X-ray intensity (enhancement) and another one of $1 / 4$ in the part of the tertiary fluorescent X-ray intensity (third-element effect) were left out as demonstrated by Shiraiwa and Fujino [18]. Of course, these missing factors have now been added to the initial Sherman equation. This equation is of vital importance in $\mathrm{XRF}$ analysis for two main reasons. Firstly, it enables us to calculate what we measure: line intensities. This feature is unique to X-ray spectrometry. No other analytical technique allows for such a combination of theoretical physics and experimental results. Secondly, the Sherman equation can provide the theoretical basis of all modern models for the correction of matrix effects [1].

The complex Sherman equation can be rewritten more simply, after some algebraic manipulations, in the following mathematical form [1]:

$$
R_{i}=C_{i} \frac{1+\sum_{j} \varepsilon_{i j} C_{j}}{1+\sum_{j} \alpha_{i j} C_{j}}
$$

where

$$
\begin{aligned}
\varepsilon_{\mathrm{ij}} & =\frac{\sum_{\mathrm{k}} \mathrm{W}_{\mathrm{i}}\left(\lambda_{\mathrm{k}}\right) \delta_{\mathrm{ij}}\left(\lambda_{\mathrm{k}}\right)}{\sum_{\mathrm{k}} \mathrm{W}_{\mathrm{i}}\left(\lambda_{\mathrm{k}}\right)} \\
\alpha_{\mathrm{ij}} & =\frac{\sum_{\mathrm{k}} \mathrm{W}_{\mathrm{i}}\left(\lambda_{\mathrm{k}}\right) \beta_{\mathrm{ij}}\left(\lambda_{\mathrm{k}}\right)}{\sum_{\mathrm{k}} \mathrm{W}_{\mathrm{i}}\left(\lambda_{\mathrm{k}}\right)}
\end{aligned}
$$

The weighting factor, $\mathrm{W}_{\mathrm{i}}\left(\lambda_{\mathrm{k}}\right)$, is defined by

$$
\mathrm{W}_{\mathrm{i}}\left(\lambda_{\mathrm{k}}\right)=\frac{\mu_{\mathrm{i}}\left(\lambda_{\mathrm{k}}\right) \cdot \mathrm{I}_{\mathrm{o}}\left(\lambda_{\mathrm{k}}\right) \cdot \Delta \lambda_{\mathrm{k}}}{\mu_{\mathrm{i}}^{*} \cdot\left[1+\sum_{\mathrm{j}} \mathrm{C}_{\mathrm{j}} \beta_{\mathrm{ij}}\left(\lambda_{\mathrm{k}}\right)\right]}
$$

Eq. (3) is still the same Sherman equation as before, except that the absolute intensity $\left(\mathrm{I}_{\mathrm{i}}\right)$ has been replaced by the relative one $\left(\mathrm{R}_{\mathrm{i}}\right)$ simply to allow the calculated intensities to be independent of the instrument. In this form, the Sherman equation still shows that the relative intensity $R_{i}$ is proportional to the concentration $\mathrm{C}_{\mathrm{i}}$ but also to the ratio on the right-hand side of it. The numerator contains all the enhancement coefficients $\delta_{\mathrm{ij}}$ (or $\varepsilon_{\mathrm{ij}}$ ) of each element $\mathrm{j}$ of the matrix, at concentration $\mathrm{C}_{\mathrm{j}}$, and the denominator contains all the absorption coefficients $\beta_{\mathrm{ij}}$ (or $\alpha_{\mathrm{ij}}$ ) of each element $\mathrm{j}$. Thus, $R_{i}$ will increase with the enhancement effects and decrease with the absorption effects (if $\beta_{\mathrm{ij}}$ is positive). Furthermore, all these matrix effects are weighted by the factor $\mathrm{W}_{\mathrm{i}}\left(\lambda_{\mathrm{k}}\right)$, which takes into account the polychromaticity of the incident spectrum and the matrix composition of the specimen. For the identification of the other undefined variables, the reader is referred to Ref. [1].

Since in practice XRF analysts are interested in calculating concentrations rather than intensities, which are measured, Eq. (3) must be reversed in order to obtain an equation enabling us to calculate the composition of the samples to be analyzed. Thus, we obtain 


$$
\mathrm{C}_{\mathrm{i}}=\mathrm{R}_{\mathrm{i}} \cdot \frac{1+\sum_{\mathrm{j}} \alpha_{\mathrm{ij}} \mathrm{C}_{\mathrm{j}}}{1+\sum_{\mathrm{j}} \varepsilon_{\mathrm{ij}} \mathrm{C}_{\mathrm{j}}}
$$

or

$$
\mathrm{C}_{\mathrm{i}}=\mathrm{R}_{\mathrm{i}} \cdot \mathrm{M}_{\mathrm{is}}
$$

with

$$
\mathrm{M}_{\mathrm{is}}=\frac{1+\sum_{\mathrm{j}} \alpha_{\mathrm{ij}} \mathrm{C}_{\mathrm{j}}}{1+\sum_{\mathrm{j}} \varepsilon_{\mathrm{ij}} \mathrm{C}_{\mathrm{j}}}
$$

where the $\mathrm{M}_{\mathrm{is}}$ factor is the correction factor for the matrix effects of specimen "s" on analyte $i$. In practice, the $\mathrm{R}_{\mathrm{i}}$ intensities are measured and the $\mathrm{M}_{\mathrm{is}}$ factor is calculated from the Sherman equation or any theoretically valid algorithm [4] selected by the analyst for a given analytical context. For the calculation of concentrations from the measured intensities, an accurate value of $\mathrm{M}_{\text {is }}$ is needed for each unknown sample. However, $\mathrm{M}_{\mathrm{is}}$ is strongly dependent on the sample composition, which is obviously unknown prior to the analysis. Consequently, we are trapped in a vicious circle... We will see later the different solutions proposed to solve this problem in practice.

\section{PHYSICAL INTERPRETATION}

The physical interpretation of the Fundamental Algorithm (Eq. 7) is quite simple and elegant. To a first approximation, equation (7) reveals that the concentration of the analyte $i, C_{i}$, is proportional to its measured relative intensity, $\mathrm{R}_{\mathrm{i}}$, which is multiplied by a ratio correcting for all matrix effects.

The numerator contains all the absorption coefficients $\alpha_{i j}$ of each element $\mathrm{j}$ of the matrix. Thus, the numerator corrects for all absorption effects of the matrix on the analyte $i$, each element $\mathrm{j}$ bringing its contribution to the total correction in a proportion $\mathrm{C}_{\mathrm{j}}$. If the numerator is greater than unity (it could be lower if the matrix is less absorbent than the analyte), the intensity $R_{i}$ will be increased by a quantity equivalent to that absorbed by the matrix.

The denominator contains all the enhancement coefficients $\varepsilon_{\mathrm{ij}}$ of each element $\mathrm{j}$ of the matrix. If some elements $\mathrm{j}$ are able to enhance the analyte $i$, the corresponding coefficient $\varepsilon_{\mathrm{ij}}$ will be different from zero and always positive. Thus, the denominator corrects for all enhancement effects of the matrix on the analyte $i$, each element $\mathrm{j}$ bringing its contribution to the total correction in a proportion $C_{j}$. In the case of enhancement, the denominator will be greater than unity and the intensity $R_{i}$ will be reduced by a quantity equivalent to that caused by the enhancement. Thus, the intensity $R_{i}$ in the equation (7) will increase with the absorption effects (if the term $\Sigma_{j} \alpha_{i j} C_{j}$ is positive) and decrease with the enhancement effects.

Since the numerator of the Fundamental Algorithm corrects for all absorption effects of the matrix on the analyte and since the denominator corrects for all enhancement effects, the form of this equation makes it easier to understand the physical principles behind the complex equation of Sherman.
In physics, the validity of a new theory is confirmed if it reveals new facts. Regarding the FA, it reveals that the coefficients $\alpha_{i j}$ and $\varepsilon_{i j}$ are the weighted means of all absorption and enhancement effects, respectively, caused by element $j$ on analyte $\mathrm{i}$, where to each incident wavelength $\lambda_{\mathrm{k}}$ is given a weight $\mathrm{W}_{\mathrm{i}}$, which takes into account the polychromaticity of the incident spectrum [1].

The knowledgeable reader may ask why I have not retained the canonical form of the algorithm proposed by Broll-Tertian [19] or Lachance-Claisse [20] rather than the one of the FA? These two algorithms have in common the fact that they merge in one new coefficient the coefficients for the correction of absorption and enhancement effects. By doing it, the above algorithms do not describe properly, or distort, physical reality as described by the Sherman equation $[21,22]$. Such is not the case with the FA, which completely respects the Sherman equation.

\section{A PHYSICAL DEMONSTRATION OF THE FUN- DAMENTAL ALGORITHM}

A more "physical" demonstration of the Fundamental Algorithm is as follows. In 1966, Lachance and Traill [6] proposed the following definition of the absorption influence coefficient for a monochromatic incident source of wavelength $\lambda_{\mathrm{k}}$ :

$\beta_{\mathrm{ij}}\left(\lambda_{\mathrm{k}}\right)=\frac{\mu_{\mathrm{j}}^{*}}{\mu_{\mathrm{i}}^{*}}-1$

where

$\mu_{j}^{*}=\mu_{j}\left(\lambda_{k}\right) \operatorname{cosec} \phi^{\prime}+\mu_{j}\left(\lambda_{i}\right) \operatorname{cosec} \phi^{\prime \prime}$

$\mu_{\mathrm{i}}^{*}=\mu_{\mathrm{i}}\left(\lambda_{\mathrm{k}}\right) \operatorname{cosec} \phi^{\prime}+\mu_{\mathrm{i}}\left(\lambda_{\mathrm{i}}\right) \operatorname{cosec} \phi^{\prime \prime}$

The $\beta_{\mathrm{ij}}\left(\lambda_{\mathrm{k}}\right)$ coefficient corrects for the absorption effect of the matrix element $\mathrm{j}$ on the analyte $\mathrm{i}$ when the incident X-ray source is monochromatic [1]. In 1984, Rousseau [16] generalized this definition for a polychromatic incident source:

$\alpha_{\mathrm{ij}}=\frac{\sum_{\mathrm{k}} \mathrm{w}_{\mathrm{i}}\left(\lambda_{\mathrm{k}}\right) \beta_{\mathrm{ij}}\left(\lambda_{\mathrm{k}}\right)}{\sum_{\mathrm{k}} \mathrm{w}_{\mathrm{i}}\left(\lambda_{\mathrm{k}}\right)}$

where

$\mathrm{W}_{\mathrm{i}}\left(\lambda_{\mathrm{k}}\right)=\frac{\mu_{\mathrm{i}}\left(\lambda_{\mathrm{k}}\right)}{\mu_{\mathrm{i}}^{*}} \cdot \frac{\mathrm{I}_{\mathrm{O}}\left(\lambda_{\mathrm{k}}\right) \Delta \lambda_{\mathrm{k}}}{1+\sum_{\mathrm{j}} \mathrm{C}_{\mathrm{j}} \beta_{\mathrm{ij}}\left(\lambda_{\mathrm{k}}\right)}$

is the weighting factor of each wavelength $\lambda_{\mathrm{k}}$ of the incident $\mathrm{X}$-ray spectrum. The $\alpha_{\mathrm{ij}}$ coefficient is the weighted mean of all the absorption effects caused by matrix element $\mathrm{j}$ on analyte $\mathrm{i}$ in a given specimen when it is bombarded by a polychromatic incident spectrum. The value of the $\alpha_{i j}$ coefficient is unique and fundamental for a given set of experimental conditions and for a given specimen composition. Note that the coefficient $\beta_{\mathrm{ij}}\left(\lambda_{\mathrm{k}}\right)$ is a binary coefficient depending only on elements $i$ and $j$. On the other hand, the coefficient $\alpha_{i j}$ is a multi-element coefficient depending on the full matrix composition including element $\mathrm{j}$. 
If there is no enhancement, the concentration $C_{i}$ is calculated by the following algorithm:

$\mathrm{C}_{\mathrm{i}}=\mathrm{R}_{\mathrm{i}}\left(1+\sum_{\mathrm{j}} \alpha_{\mathrm{ij}} \mathrm{C}_{\mathrm{j}}\right)$

which has the same form as the Lachance-Traill algorithm [6]. In the general case where both matrix effects, absorption and enhancement, are present, this latter algorithm becomes

$\mathrm{C}_{\mathrm{i}}=\mathrm{R}_{\mathrm{i}} \frac{1+\sum_{\mathrm{j}} \alpha_{\mathrm{ij}} \mathrm{C}_{\mathrm{j}}}{1+\sum_{\mathrm{j}} \varepsilon_{\mathrm{ij}} \mathrm{C}_{\mathrm{j}}}$

which is the Fundamental Algorithm where

$\varepsilon_{i j}=\frac{\sum_{k} W_{i}\left(\lambda_{k}\right) \delta_{i j}\left(\lambda_{k}\right)}{\sum_{k} W_{i}\left(\lambda_{k}\right)}$

The $\varepsilon_{\mathrm{ij}}$ coefficient is the weighted mean of all the enhancement effects caused by matrix element $j$ on analyte $i$ in a given specimen when it is bombarded by a polychromatic incident spectrum. The value of the $\varepsilon_{\mathrm{ij}}$ coefficient is unique and fundamental for a given set of experimental conditions and for a given specimen composition.

As demonstrated, the Fundamental Algorithm is the generalization of the Lachance-Traill algorithm when the specimen is bombarded by a polychromatic incident spectrum and where both matrix effects, absorption and enhancement, are present. The $\alpha_{i j}$ and $\varepsilon_{i j}$ coefficients are multi-element coefficients depending on the full matrix composition of each specimen. We will see now how to calculate them for each sample to be analyzed.

\section{THE PRACTICAL APPLICATION OF THE FUN- DAMENTAL ALGORITHM}

When a series of values of the $\alpha_{i j}$ and $\varepsilon_{i j}$ coefficients is calculated for a given specimen, these values are valid only for this specific specimen since they depend on the full matrix composition. Any other specimen with the same series of elements, but in different proportions, will need a new set of coefficient values to correct accurately for matrix effects.

Since the $\alpha_{\mathrm{ij}}$ and $\varepsilon_{\mathrm{ij}}$ coefficients depend on the total matrix composition, the composition of each sample must be first calculated from an initial estimate of the composition. It is calculated using the Claisse-Quintin algorithm [7]:

$$
\begin{aligned}
C_{i}=R_{i}\left[1+\sum_{j}\left(a_{i j}+a_{i j j} C_{M}\right) C_{j}\right. \\
\left.+\sum_{j} \sum_{k>j} a_{i j k} C_{j} C_{k}\right]
\end{aligned}
$$

where $C_{M}$ is the concentration of the total matrix, $a_{i j}$ and $a_{i j j}$ are binary, and $\mathrm{a}_{\mathrm{ijk}}$, ternary influence coefficients. Then, from this estimated composition, all $\alpha_{\mathrm{ij}}$ and $\varepsilon_{\mathrm{ij}}$ coefficients, the complex part of Sherman's equation, are calculated once only. With these calculated coefficients now used as constants, the final (and more accurate) composition of the sample is calculated by applying an iteration process to the Fundamental Algorithm.
To apply this method in practice, a commercial WINDOWS ${ }^{\text {TM }}$ software package known as CiROU is available [23].

The Fundamental Algorithm method has the following clear advantages:

1. Since the numerator of equation (7) corrects for all the absorption effects of the matrix on the analyte and since the denominator corrects for all the enhancement effects, the form of equation (7) makes it much easier to understand the physical principles behind the complex equation of Sherman. Consequently, its great beauty lies in its perfect symmetry.

2. For the first time, it enabled to deduce the concept of influence coefficients directly from the Sherman equation without any approximation. It proposes the fundamental, unique and explicit equations for calculating the $\alpha_{\mathrm{ij}}, \varepsilon_{\mathrm{ij}}$ coefficients, only in terms of fundamental parameters.

3. The equations of the $\alpha_{\mathrm{ij}}, \varepsilon_{\mathrm{ij}}$ coefficients reveal that they are the weighted means of all absorption and enhancement effects, respectively, caused by element $\mathrm{j}$ on analyte $i$ in a given specimen. They also introduce a weighting factor, $\mathrm{W}_{\mathrm{i}}$, for each incident wavelength $\lambda_{\mathrm{k}}$ of the incident spectrum.

4. Empirical coefficients are no longer required. The Fundamental Algorithm uses only theoretical influence coefficients that are in full agreement with the treatment of physics as proposed by Sherman. They are calculated for each sample composition, increasing the accuracy in so doing.

5. This method also uses a fully theoretical approach to calculate all the required parameters. For example, the method uses equations proposed by Pella et al. [17] to calculate up to 350 different intensities of the incident spectrum emitted by the X-ray tube. It uses data from Heinrich [24] to calculate mass absorption coefficients by a method proposed by Springer and Nolan [25]. It also uses modern values of X-ray fluorescence yields [26, 27] and Coster-Kronig transition probabilities [28].

6. The method can be used in practice to calculate the composition of any sample type, of any composition, i.e., for concentration ranges varying from 0 to $100 \%$. It introduces a theoretical mean relative error of $0.05 \%$ only [4]. An experimental verification of this method done by Rousseau and Bouchard [29] on different types of alloy has confirmed its accuracy and versatility.

7. In contrast to the Fundamental-Parameters method, it directly calculates concentrations rather than intensities, and the calculation technique has been optimized.

8. Normalization of calculated concentrations is no longer required. Since the complex part of Sherman's equation is calculated once only, the calculation time is greatly reduced.

9. It allows deduction of other theoretically valid algorithms, such as the Lachance-Traill or the Claisse- 
Quintin algorithm [1]. In other words, it can be the source of all modern methods to correct for matrix effects.

10. It takes advantage of 30 years of research and development on mathematical models for matrix effect corrections (see Section 2).

However, this theoretical approach needs to be adapted to the experimental data of each spectrometer, since theory cannot account for all the instrumental parameters. It is done through a smart calibration procedure that compares the measured intensities to the calculated ones [2].

\section{THEORETICAL BINARY INFLUENCE COEFFI- CIENTS}

In quantitative XRF analysis, one of the major problems is the correction for matrix effects (absorption and enhancement). The Fundamental Algorithm uses theoretical multielement influence coefficients, which are numerical coefficients that correct for the effect of each matrix element on the element to be determined (or analyte) in a given specimen. However, these coefficients depend on the full sample composition to be analyzed (see Eqns 4 and 5), which in practice is unknown prior to analysis and they must be calculated for each sample as already explained. One of the solutions proposed to solve this problem was the concept of theoretical binary influence coefficients.

These binary coefficients are based on the hypothesis that the total matrix effect on the analyte $i$ is equal to the sum of the effects of each element $j$ of the matrix, each of these effects being calculated independently of each other. In other words, from a practical point of view, it is easier to consider a sample as a sum of binary mixtures rather than as a multielement mixture. Of course, this approach is an approximation because one cannot isolate the matrix effect of each element $j$ on the analyte $i$ from the effect of the rest of the matrix. But this approach allows one to correct for matrix effects with accuracy as long as the composition range of samples to analyze is fairly limited. With this approach it was then possible to calculate a set of theoretical binary influence coefficients valid for a given composition range rather than for a given sample. In other words, with binary coefficients it is assumed that the binary coefficient $a_{i j}$ is a constant for a given range of $C_{i}$ and $C_{j}$ rather than being a variable dependent on the whole matrix composition of each sample.

Theoretical binary influence coefficients can be calculated by using the Fundamental Algorithm. With this equation, the intensities emitted by representative binary standards are calculated rather than being measured. With this approach, one assumes that the composition of a complex sample is made up of a series of binary elements or compounds where one considers the effect of one matrix element at a time on each analyte, independently of the rest of the matrix composition. Thus, a series of influence coefficients is calculated from hypothetical compositions for the binary series of elements or compounds that are present in the samples.

Two different algorithms can use the modern concept of theoretical binary influence coefficients. These two algorithms, among all the proposed ones, have been chosen because of their accuracy and their sound theoretical basis. They are:
First, the Lachance-Traill (LT) algorithm [6]:

$$
\mathrm{C}_{\mathrm{i}}=\mathrm{R}_{\mathrm{i}}\left(1+\sum_{\mathrm{j}} \mathrm{a}_{\mathrm{ij}} \mathrm{C}_{\mathrm{j}}\right)
$$

where $R_{i}$ is the ratio of the measured net intensity $I_{i}$ to the measured net intensity of the pure analyte $i$. The binary coefficient $\mathrm{a}_{\mathrm{ij}}$ is calculated using the following equation [1]:

$$
a_{i j}=\frac{\alpha_{i j}-\varepsilon_{i j}}{1+\varepsilon_{i j} C_{j m}}
$$

where $\alpha_{\mathrm{ij}}$ and $\varepsilon_{\mathrm{ij}}$, defined by equations (5) and (4), are calculated for the special case of a binary standard having a composition $\left(\mathrm{C}_{\mathrm{im}}, \mathrm{C}_{\mathrm{jm}}\right)$, where $\mathrm{C}_{\mathrm{im}}$ is the mid-value of the calibration range of the analyte $i$ and where

$$
\mathrm{C}_{\mathrm{jm}}=1-\mathrm{C}_{\mathrm{im}}
$$

Nowadays, the alpha coefficients as proposed by Lachance and Traill [6] are no longer used in practice because of their lack of accuracy. They have been replaced by the theoretical binary influence coefficients $\mathrm{a}_{\mathrm{ij}}$, which, as opposed to the alpha coefficients, take into account the enhancement effect as well as the polychromaticity of the incident radiation [1].

This approach assumes that the coefficient $\mathrm{a}_{\mathrm{ij}}$ is a constant (it is an approximation!) when it is applied to specimens with a limited concentration range $(0-10 \%)$, such as, for example, oxides in rock samples diluted in fused discs. In this case, the calculation method by itself (Eqns 19, 20 and 21) introduces a theoretical mean relative error of only $0.02 \%$ on the calculated concentrations. On the other hand, for concentration variations greater than $10 \%$, the concentrations calculated by this algorithm associated to the $\mathrm{a}_{\mathrm{ij}}$ coefficients are unacceptable [4]. See the experimental verification at the end of this paper.

In the case of diluted samples such as fused discs or pressed powder pellets, the theoretical binary influence coefficient $\left(a_{i j}\right)$ defined above can be modified by incorporating a constant term. For example, when a sample is fused in a fixed sample/flux ratio to produce a fused disc, or when a pulverized sample is mixed in a fixed sample/binder ratio and pressed, the $\mathrm{a}_{\mathrm{ij}}$ coefficient can be modified by including the weight fraction and the composition of the flux or the binder, which are essentially constant for every specimen. In this case, the $\mathrm{a}_{\mathrm{ij}}$ coefficient is referred to as a modified coefficient. The coefficients $\mathrm{a}_{\mathrm{ij}}$ can also be modified to express them in terms of oxides rather than elements themselves.

Two different $\mathrm{a}_{\mathrm{ij}}$ coefficients, calculated for the correction of matrix effects of two different matrix elements on the analyte, can be combined to form only one coefficient. In this case, the new coefficient is referred to as a hybrid coefficient. It is an elegant way to eliminate the measurement of one analyte and to correct for its matrix effects even if it has not been measured or its concentration is not known. However, this approach introduces more approximations and must be used with caution and applied with great care. The terminology "modified" and "hybrid" influence coefficients has been proposed by Lachance [30] in 1979 but his methods of calculation have not been retained. 
When samples are prepared as fused discs, volatile products (e.g. $\mathrm{CO}_{2}, \mathrm{H}_{2} \mathrm{O}, \mathrm{SO}_{2}, \mathrm{Cl}, \mathrm{F}$, etc.) can be lost during the fusion and/or it can be accompanied with a gain in weight due to oxidation (e.g. $\mathrm{FeO} \rightarrow \mathrm{Fe}_{2} \mathrm{O}_{3}$ ). In this case, there are three different ways to calculate the sample composition:

1. A conventional Loss On Ignition (LOI) is done on the pulverized sample BEFORE the fusion and the ignited powder is used to prepare the fused disc. In this case, there is usually NO further loss of volatile or gain in weight during the fusion and all the calculated concentrations in the fused disc are adjusted to take into account the LOI and get the analyte concentrations in the sample [31]. This approach generates accurate results except it is time consuming.

2. A conventional LOI is done on the pulverized sample, but the original sample is used to prepare the fused disc. In this case, there is loss of volatile and/or gain in weight during the fusion. It changes the sample/flux ratio and may severely both affects the accuracy of results. The method developed by us takes this phenomenon into account by using a theoretical approach based on the famous Sherman equation [31]. The coefficient thus calculated for the loss of volatile products and/or gain in weight is included in the term $[1+\ldots]$ correcting for matrix effects, even if it is not an influence coefficient. This approach generates accurate results and it is less time consuming than the previous one because the LOI and the fused disc can be done at the same time.

3. No conventional LOI is done on the pulverized sample. In this case, there is loss of volatile and/or gain in weight during the fusion and the LOI value is unknown. The LOI value is calculated by difference between $100 \%$ and the sum of calculated concentrations in the sample [31]. Knowing the LOI value, the sample composition is recalculated as in the previous case. This approach introduces more approximations in the calculation method and is sensitive to any experimental errors. Since the accuracy of this approach is more "unpredictable", it must be applied with caution and great care.

Second, the Claisse-Quintin (CQ) algorithm [7,13,14,29]:

The Claisse-Quintin algorithm (CQ) can be described as an extension of the Lachance-Traill algorithm (LT) taking into account the fact that the LT coefficient, $\mathrm{a}_{\mathrm{ij}}{ }^{\mathrm{LT}}$ (see Eq. 22), is not a constant but varies with the concentration of the matrix elements. According to Claisse and Quintin the LT coefficient $\mathrm{a}_{\mathrm{ij}}{ }^{\mathrm{LT}}$ varies linearly with the concentration $C_{j}$, i.e.

$a_{i j}^{L T}=a_{i j}+a_{i j j} C_{j}$

Thus, the general form of the Claisse-Quintin algorithm for a multicomponent sample can be written as:

$$
\begin{aligned}
C_{i}=R_{i} \cdot\left[1+\sum_{j}\left(a_{i j}\right.\right. & \left.+a_{i j j} C_{M}\right) C_{j} \\
& \left.+\sum_{j} \sum_{k>j} a_{i j k} C_{j} C_{k}\right]
\end{aligned}
$$

where the matrix concentration $\mathrm{C}_{\mathrm{M}}$ is the sum of all elements in the sample except i, i.e.

$$
\mathrm{C}_{\mathrm{M}}=1-\mathrm{C}_{\mathrm{i}}=\mathrm{C}_{\mathrm{j}}+\mathrm{C}_{\mathrm{k}}+\ldots+\mathrm{C}_{\mathrm{N}}
$$

and where the "crossed" ternary coefficient $a_{i j k}$ has been added to compensate for the fact that the total matrix correction cannot be strictly represented by a weighted sum of binary corrections. The binary coefficients $\mathrm{a}_{\mathrm{ij}}$ and $\mathrm{a}_{\mathrm{ijj}}$ are calculated from theory at hypothetical binary compositions of $\left(\mathrm{C}_{\mathrm{i}}, \mathrm{C}_{\mathrm{j}}\right)=(0.2,0.8)$ and $(0.8,0.2)$, respectively. The crossproduct coefficient, $\mathrm{a}_{\mathrm{ijk}}$, is calculated at the ternary composition of $\left(\mathrm{C}_{\mathrm{i}}, \mathrm{C}_{\mathrm{j}}, \mathrm{C}_{\mathrm{k}}\right)=(0.30,0.35,0.35)$. To be more explicit [32], if for a ternary system $\left(C_{i}, C_{j}, C_{k}\right)$, the variable $F_{i}\left(C_{i}\right.$, $\mathrm{C}_{\mathrm{j}}, \mathrm{C}_{\mathrm{k}}$ ) is defined by

$\mathrm{F}_{\mathrm{i}}\left(\mathrm{C}_{\mathrm{i}}, \mathrm{C}_{\mathrm{j}}, \mathrm{C}_{\mathrm{k}}\right)=\frac{1}{\mathrm{C}_{\mathrm{j}}}\left(\frac{\mathrm{C}_{\mathrm{i}}}{\mathrm{R}_{\mathrm{i}}}-1\right)$

Note that: if $C_{j}=0$, then

$\mathrm{F}_{\mathrm{i}}\left(\mathrm{C}_{\mathrm{i}}, 0, \mathrm{C}_{\mathrm{k}}\right)=\frac{1}{\mathrm{C}_{\mathrm{k}}}\left(\frac{\mathrm{C}_{\mathrm{i}}}{\mathrm{R}_{\mathrm{i}}}-1\right)$

where the ratio $\mathrm{C}_{\mathrm{i}} / \mathrm{R}_{\mathrm{i}}$ is calculated by the Fundamental Algorithm (Eq. 7) for a ternary system:

$\frac{C_{i}}{R_{i}}=\frac{1+\alpha_{i j} C_{j}+\alpha_{i k} C_{k}}{1+\varepsilon_{i j} C_{j}+\varepsilon_{i k} C_{k}}$

The three coefficients of the CQ algorithm are calculated by means of:

$$
\begin{aligned}
& \mathrm{a}_{\mathrm{ij}}=\frac{1}{3}\left[-\mathrm{F}_{\mathrm{i}}(0.2,0.8,0)+4 \mathrm{~F}_{\mathrm{i}}(0.8,0.2,0)\right] \\
& \mathrm{a}_{\mathrm{ijj}}=\frac{5}{3}\left[\mathrm{~F}_{\mathrm{i}}(0.2,0.8,0)-\mathrm{F}_{\mathrm{i}}(0.8,0.2,0)\right] \\
& \mathrm{a}_{\mathrm{ijk}}=\frac{20}{7}\left[\begin{array}{l}
\mathrm{F}_{\mathrm{i}}(0.3,0.35,0.35) \\
-\mathrm{F}_{\mathrm{i}}(0.3,0.7,0) \\
-\mathrm{F}_{\mathrm{i}}(0.3,0,0.7)
\end{array}\right]
\end{aligned}
$$

The influence coefficients of the CQ algorithm are considered as constants when they are applied to specimens with a medium concentration range $(0-40 \%)$, such as, for example, oxides in cement samples in pressed pellets. In this case, the calculation method by itself introduces a theoretical mean relative error of $0.04 \%$ on the calculated concentrations [4].

As with the LT algorithm, when a pulverized sample is mixed in a fixed sample/binder ratio and pressed, the influence coefficients of the $\mathrm{CQ}$ algorithm can be modified by including the weight fraction and the composition of the binder, which are essentially constant for every specimen. The influence coefficients of this algorithm can also be modified to express them in terms of oxides rather than elements themselves. The hybrid coefficients are not calculated for the CQ algorithm because there is no LOI (or gain in weight) during the preparation of pressed pellets. 
The experimental verification of these two algorithms (see Section 10) confirms the expected theoretical accuracy. Consequently, XRF analysts should consider the theoretical binary coefficient approach within the LT and CQ algorithms as a valuable alternative to the FundamentalParameters approach, especially when the variations of matrix effects or composition of samples to analyze are small. For the calibration and calculation of sample compositions using the two presented algorithms with their associated theoretical influence coefficients, some commercial WINDOWS ${ }^{\mathrm{TM}}$ software packages, such as CiLT and CiROU, running only on PC, are available [23].

\section{CALIBRATION}

Calibration is a very important part of any solution to any FP method because it takes into account the imperfections of theory and experimental errors. Indeed, as pointed out by Criss [33], many assumptions are made in order to be able to use the Sherman equation in practice. For example, the geometry of the specimen excitation is oversimplified: The exit angle from the tube target and the incident angle of the primary radiation over the surface of the specimen vary widely; nevertheless, both parameters are represented by a single number. One also assumes that the primary radiation is parallel, and that the X-rays travel effectively in a straight line within the specimen until they are absorbed. Moreover, only one level of enhancement is taken into account, and it is assumed that there is no scattering of X-ray fluorescence intensities and that all measured fluorescence X-rays exit the specimen at the same angle. There are many reasons to doubt the accuracy of the absorption and enhancement corrections: Mass absorption coefficients are only known to about $1 \%$; in calculating the corrections, and the total attenuation coefficient is often used as an approximation for the photoelectric coefficient.

Finally, there are many effects that the model does not address at all. For example, it does not account for the X-ray tube current, various solid angles, the reflectivity of the analyzing crystal and the detector efficiency. The $1 / \mathrm{r}^{2}$ dependence of intensity on distance from the target is also ignored. In conclusion, the Sherman equation neglects several types of interactions. Clearly, the mathematical model proposed by Sherman is imperfect, but no model can account totally for all the subtleties of instrument response and X-ray interactions within the specimen. Nevertheless, experience shows that the model performs very well in practice [29] if all of these approximations are compensated by using an appropriate calibration procedure [3], as illustrated below. Willy de Jongh [11] was the first in 1973 to propose the philosophy of this calibration procedure that compares measured intensities to calculated intensities (he called them unrealistically "apparent concentrations"). However, the author proposes the same thing today from a completely new approach.

\section{CALIBRATION AND DRIFT MONITORS}

Although in many cases the analysis of samples with a limited composition range may allow the use of empirical calibration curves comparing uncorrected (for matrix effects) net intensities to concentrations, it is usually preferable to work with a general-purpose calibration procedure that is applicable to a larger variety of matrix types covering wide concentration ranges. Furthermore, with theoretical FP mod- els (such as the FA), a special calibration procedure must be used, because it is well known that any theory cannot account for the variations of all instrumental parameters. To reach these two objectives, the following calibration procedure is proposed.

Recalling that the relative intensity $R_{i}$ is defined as the ratio of the net intensity $I_{i}$ of the analyte $i$ to the net intensity $\mathrm{I}_{(\mathrm{i})}$ of the pure analyte $\mathrm{i}$ :

$\mathrm{R}_{\mathrm{i}}=\frac{\mathrm{I}_{\mathrm{i}}}{\mathrm{I}_{(\mathrm{i})}}$

This simple equation can be rewritten in the following form:

$\mathrm{I}_{\mathrm{i}}=\mathrm{I}_{(\mathrm{i})} \mathrm{R}_{\mathrm{i}}$

Dividing both sides of this equation by $\mathrm{I}_{\mathrm{MM}}$, the measured gross intensity of the analyte $\mathrm{i}$ in any drift monitor, leads to

$\frac{\mathrm{I}_{\mathrm{i}}}{\mathrm{I}_{\mathrm{iM}}}=\frac{\mathrm{I}_{(\mathrm{i})}}{\mathrm{I}_{\mathrm{iM}}} \cdot \mathrm{R}_{\mathrm{i}}$

This simple mathematical operation allows to correct any measured intensity for the instrumental drift. Indeed, even if the absolute value of intensities $\mathrm{I}_{\mathrm{i}}$ and $\mathrm{I}_{\mathrm{iM}}$ vary because of the instrumental drift, their ratio remains constant [3].

The drift monitor is any type of stable specimen containing a statistically significant concentration of one or several analytes. The drift monitor intensities for the various analytes must be in the linear range of the counting electronics and high enough so that counting errors are minimized within reasonable counting times for each element (e.g. 60 s). In practice, it is recommended that the drift monitor intensity of each analyte is slightly higher than the highest intensity of the analyte concentration range. When a single drift monitor specimen does not contain all the elements to be determined in the samples, or when it is not possible to measure statistically significant intensity values for all analytes from a single drift monitor specimen, the use of several drift monitor specimens is required. The matrix composition of a drift monitor may be completely different from that of the samples to be analyzed. For example, for the analysis of rock samples, a good drift monitor can be a vitrified synthetic specimen, a glass, an alloy, a selected standard or any other sample that is completely different physically from the samples to be analyzed. If available, pure element specimens can be used for every high-concentration analyte. Otherwise, for each analyte, a selected unknown sample containing the highest concentration of the considered analyte is used. A drift monitor does not need to be (but preferably should be) homogeneous and infinitely thick. However, it must have a flat and polished (without grooves) surface for high reproducibility. Since it is used solely as an intensity reference to evaluate the instrumental drift, its exact composition does not need to be known, provided that it remains stable with time. Finally, the measured intensity $\mathrm{I}_{\mathrm{iM}}$ of the analyte $\mathrm{i}$ in the drift monitor does not need to be corrected for background, line overlaps, blank, etc., since it is always measured under the same experimental conditions. 

i.e.

Eq. (33) has the general form of a straight-line equation,

$$
\mathrm{Y}_{\mathrm{i}}=\mathrm{m}_{\mathrm{i}} \mathrm{X}_{\mathrm{i}}
$$

where $m_{i}$ is the slope of the line. If we plot a calibration line of the measured relative intensity $\mathrm{I}_{\mathrm{i}} / \mathrm{I}_{\mathrm{iM}}$ (Y-axis) as a function of the calculated (or theoretical) relative intensity $\mathrm{R}_{\mathrm{i}}(\mathrm{X}$ axis), the slope $m_{i}$ of the line is equal to

$\mathrm{m}_{\mathrm{i}}=\frac{\mathrm{I}_{(\mathrm{i})}}{\mathrm{I}_{\mathrm{iM}}}$

The theoretical relative intensity $R_{i}$ can be calculated from Eq. (8) rewritten in the following form:

$$
\mathrm{R}_{\mathrm{i}}=\frac{\mathrm{C}_{\mathrm{i}}}{\mathrm{M}_{\mathrm{is}}}
$$

The $\mathrm{M}_{\mathrm{is}}$ factor, that corrects for matrix effects, can be calculated from the Sherman equation or any theoretically valid algorithm between $C_{i}$ and $R_{i}$ [4], for example, the one of Lachance-Traill or Claisse-Quintin or the Fundamental Algorithm, selected by the analyst for the given type of samples to be analyzed. The $R_{i}$ intensities are calculated for the composition of all the standards at hand and their $\mathrm{I}_{i}$ intensities, and also the $\mathrm{I}_{\mathrm{iM}}$ intensities, are measured.

The combination of (33), (35) and (36) leads to

$$
\frac{\mathrm{I}_{\mathrm{i}}}{\mathrm{I}_{\mathrm{iM}}}=\mathrm{m}_{\mathrm{i}} \cdot \frac{\mathrm{C}_{\mathrm{i}}}{\mathrm{M}_{\mathrm{is}}}
$$

This linear equation does not contain an intercept value because it is assumed that the true net intensity $\mathrm{I}_{i}$ is equal to zero when $C_{i}$ is equal to zero. However, for many reasons (incorrect background, interference or blank subtraction, etc.), it may happen that $\mathrm{I}_{i}$ is different from zero even if $\mathrm{C}_{i}$ is zero. In that case, the introduction of an intercept can allow avoiding important systematic errors, especially for low concentration values. Allowing then for the presence of an intercept $b_{i}$, Eq. (37) becomes

$$
\frac{\mathrm{I}_{\mathrm{i}}}{\mathrm{I}_{\mathrm{iM}}}=\mathrm{m}_{\mathrm{i}} \cdot \frac{\mathrm{C}_{\mathrm{i}}}{\mathrm{M}_{\mathrm{is}}}+\mathrm{b}_{\mathrm{i}}
$$

which is the equation used to plot any calibration line in association with any FP model. Here, the intercept $b_{i}$ is theoretically zero, unless $I_{i}$ has not been perfectly corrected for background, line overlaps, etc. When calibrating for an analyte, it must always be kept in mind that a significant intercept value indicates the presence of systematic errors and therefore should not be tolerated unless one knows the reason for it and is ready to accept it.

Thus, we can plot a calibration line of measured net intensity of the analyte $\mathrm{i}\left(\mathrm{I}_{\mathrm{i}}\right)$, corrected for the instrumental drift by $\mathrm{I}_{\mathrm{iM}}$, as a function of the calculated ratio $\mathrm{C}_{\mathrm{i}} / \mathrm{M}_{\mathrm{is}} \cdot \mathrm{C}_{\mathrm{i}}$ is the concentration of the analyte $i$ in a standard and the term $\mathrm{M}_{\text {is }}$ is calculated from the Sherman equation [1] or any theoretically valid algorithm [4]. The slope of the line represents the net intensity of the pure element $i$ (see Eq. 35). Thus, this calibration procedure represents very well the physical reality because we are consistently comparing measured relative intensities $\left(\mathrm{I}_{\mathrm{i}} / \mathrm{I}_{\mathrm{iM}}\right)$ to calculated relative intensities $\left(R_{i}=C_{i} / M_{i s}\right)$. It is the best way to get a true calibration straight-line. This feature makes the calibration lines robust, i.e., they can be extrapolated by a factor of two or three, thus protecting the analyst from errors when the concentrations of samples to be analyzed exceed the calibration range. In addition, incorrect standards will be shown clearly as outliers in the calibration line. Fig. (1) shows an example of the type of calibration straight line represented by equation (38).

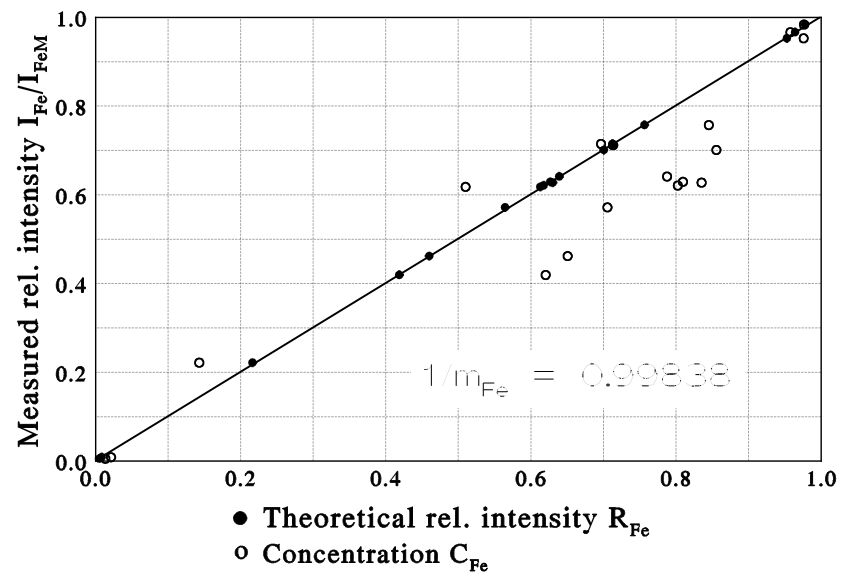

Fig. (1). Fe calibration graph using NIST alloy standards. The graph of measured relative intensities as a function of the concentration $\mathrm{C}_{\mathrm{Fe}}$ gives scattered points (o). On the other hand, the plot of the same measured relative intensities as a function of the theoretical relative intensities calculated by the Fundamental Algorithm (Eq. 7) lines up each point $(\bullet)$ on the calibration line.

The calibration slope $m_{i}$ allows the theory to be adapted to the experimental data for each spectrometer. If the pure analyte specimen is used as a drift monitor, Eq. (35) gives a slope $\mathrm{m}_{\mathrm{i}}=1.0$. If the experimental value of $\mathrm{m}_{\mathrm{i}} \neq 1.0$, the deviation results from the imperfections of the theoretical model that cannot account for all of the experimental parameters of a given spectrometer. The slope of the calibration line represents the experimental average intensity of the pure analyte determined from a set of multi-element standards. Depending on the analytical context, this measured value might be substantially different from the theoretical value. It is this difference that compensates to a large extent for all the theoretical limitations mentioned above. The observed slope value is the required factor allowing adapting theory to a particular set of experimental data and makes truly meaningful the equal sign in Eq. (38) or Eq. (39).

The above described calibration procedure is truly matrix independent because it can put on a straight line any type of matrix compositions containing the analyte. Thus, its great advantage is that it can include on the same calibration line a large variety of matrix compositions, limited only by the physical effects (particle or grain size, mineralogy, heterogeneity, surface, thickness, etc.). However, the preparation of standards must in all aspects be absolutely identical to that of the unknowns.

If empirical coefficients are used rather than theoretical ones, they are calculated during the calibration procedure by regression analysis, at the same time that the $m_{i}$ and $b_{i}$ values of all analytes. Because of the large number of unknown 
values to determine, a corresponding large number of standard specimens is needed. On the other hand, if theoretical coefficients are used, they are calculated before the calibration procedure from theory [4]. Knowing them, each calibration line requires only a minimum of two points for calculating the $m_{i}$ and $b_{i}$ values. Therefore, the great advantage of theoretical coefficients, in addition to be accurate, is that only a few standards are needed to calibrate.

This calibration procedure enables each analyte to be calibrated over wide concentration ranges and requires only a few good standards. However, for getting accuracy, it must be stressed that the complete composition of each standard is needed because the analyte line intensity is affected by the concentration of all the elements present in the specimen. If only $98 \%$ or $99 \%$ of the composition of a standard is certified, it will have limited capability for accurately calculating all the corrections for matrix effects, because the matrix composition is not well known.

Finally, one should recall that any theoretically valid model corrects only for variations in the chemical composition of the samples or standards, i.e., for elemental interactions. It does not correct for instrumental (background, line overlaps and dead time) and physical effects (variations of particle size, mineralogical, thickness and surface effects). Both must be reduced to a minimum by careful selection of measurement conditions and sample preparation procedure.

\section{ROUTINE ANALYSIS}

To apply the calibration procedure explained above in practice, rearranging Eq. (38) for $\mathrm{C}_{\mathrm{i}}$ leads to

$$
\mathrm{C}_{\mathrm{i}}=\frac{1}{\mathrm{~m}_{\mathrm{i}}} \cdot\left(\frac{\mathrm{I}_{\mathrm{i}}}{\mathrm{I}_{\mathrm{iM}}}-\mathrm{b}_{\mathrm{i}}\right) \cdot \mathrm{M}_{\mathrm{is}}
$$

The interpretation of Eq. (39) makes physical sense. Firstly, if there are some errors in the background calculation, $b_{i}$ is different from zero and subtracted from the measured net intensity $\mathrm{I}_{\mathrm{i}}$ before being corrected for matrix effects by the $\mathrm{M}_{\mathrm{is}}$ factor. Secondly, the concentration is proportional to the true net intensity, corrected for the instrumental drift (by $\mathrm{I}_{\mathrm{iM}}$ ), so that only the true net intensity is corrected for matrix effects by the $\mathrm{M}_{\mathrm{is}}$ factor. Thirdly, the measured relative intensities

$$
\mathrm{R}_{\mathrm{i}}=\frac{1}{\mathrm{~m}_{\mathrm{i}}} \cdot\left(\frac{\mathrm{I}_{\mathrm{i}}}{\mathrm{I}_{\mathrm{iM}}}-\mathrm{b}_{\mathrm{i}}\right)
$$

used with any FP model for calculating the concentrations must be corrected absolutely for the imperfections of theory by the calibration slope $\mathrm{m}_{\mathrm{i}}$ before being corrected for matrix effects.

As stressed before, the values of the slope $\left(\mathrm{m}_{\mathrm{i}}\right)$ and the intercept $\left(b_{\mathfrak{j}}\right)$ of the calibration line remain valid even after the drift of the instrument. Indeed, the slope is equal to the intensity of the pure analyte, $\mathrm{I}_{(\mathrm{i})}$, divided by the monitor intensity $\mathrm{I}_{\mathrm{iM}}$ (see Eq. 35). The intercept $b_{\mathrm{i}}$ is the interception of the calibration line with the $\mathrm{Y}$-axis and is equal to the residual background intensity divided by the monitor intensity. Thus, if the absolute values of the intensities change further to an instrumental drift, their ratio remains constant [34].
Thus, Eq. (39) is in perfect agreement with the basic physical rules of XRF spectrometry. For accurate results, it is imperative that any theoretically valid model respects the underlying physics in XRF analysis.

In routine analysis, Eq. (39) is used to calculate concentrations. A practical application of this analytical method is given in Ref. [29], where 15 different analytes were determined in many different types of alloys, covering large concentration ranges. However, the analyst must be aware of some precautions to take before using this equation:

1. When possible, and especially for trace elements, peak and background(s) should be measured for each analyte.

2. All the peak intensities should be corrected for background, line overlaps and blank, an essential step. Without effective background subtraction strategies, the accuracy of the results may be severely compromised, especially for trace elements. Background and line overlaps must be subtracted before the calibration procedure, since the concentration is proportional only to the net intensity.

3. Drift correction should be applied to the net intensity of every analyte. Only one high-point drift monitor per analyte should be used where the intensity of the analyte in the drift monitor is slightly higher than the highest measured intensity of the analyte calibration range [34]. At the same time, this procedure for drift monitors guarantees low counting statistical errors.

4. Matrix effects (by calculating the $\mathrm{M}_{\text {is }}$ factor) can be corrected using any theoretically valid model, such as the Lachance-Traill, or Claisse-Quintin or Fundamental Algorithm, in association with theoretical influence coefficients. The choice of a particular algorithm depends on the analytical context [4].

\section{EXPERIMENTAL VERIFICATION}

An application example [31] of the Fundamental Algorithm is given at Fig. (7). The concentration and the measured relative intensity data of a set of $\mathrm{Cr}-\mathrm{Fe}-\mathrm{Ni}$ specimens are given in Table 1. Figs. (3-7) compare given concentrations for $\mathrm{Fe}$ in a set of $\mathrm{Cr}-\mathrm{Fe}-\mathrm{Ni}$ specimens to the values calculated by different algorithms [4] in Fig. (2). In these figures the measured intensities are shown, respectively, uncorrected, and corrected using the Lachance-Traill (LT) algorithm (used in association with empirical and binary constant influence coefficients), the Claisse-Quintin (CQ) algorithm and the Fundamental Algorithm (FA). It can be observed that there is a progressive improvement in the fit of the data in Figs. (3-7). The data fit for the LT algorithm is not as good as the CQ or Fundamental algorithm because the Fe concentration range is too large (1-96\%). Here, the LT algorithm is used beyond its application range. Otherwise, it should give excellent results in specific contexts, such as limited concentration range $(0-10 \%)$. On the other hand, there is little improvement between the results from the CQ algorithm and the FA, which is normal. Remember that the CQ algorithm is used in association with the FA for calculating the composition of a sample. The CQ algorithm calculates an accurate first estimate of the sample composition, while the FA calculates from it the final composition. In order to make the 
FA method viable and valid, the first composition estimation must be very close to the final result. This is what we observe for these samples.

\section{Linear Algorithm}

$$
\mathrm{C}_{\mathrm{i}}=\mathrm{K}_{\mathrm{i}} \cdot \mathrm{I}_{\mathrm{i}}=\frac{1}{\mathrm{I}_{(\mathrm{i})}} \cdot \mathrm{I}_{\mathrm{i}}=\mathrm{R}_{\mathrm{i}}
$$

\section{Lachance-Traill Algorithm}

$$
\mathrm{C}_{\mathrm{i}}=\mathrm{R}_{\mathrm{i}}\left(1+\sum_{\mathrm{j}} \mathrm{a}_{\mathrm{ij}} \mathrm{C}_{\mathrm{j}}\right)
$$

\section{Claisse-Quintin Algorithm}

$$
\begin{aligned}
C_{i}=R_{i}\left[1+\sum_{j}\left(a_{i j}+a_{i j j} C_{M}\right) C_{j}\right. \\
\left.+\sum_{j} \sum_{k>j} a_{i j k} C_{j} C_{k}\right]
\end{aligned}
$$

Fundamental Algorithm (Proposed by Rousseau)

$$
C_{i}=R_{i} \frac{1+\sum_{j} \alpha_{i j} C_{j}}{1+\sum_{j} \varepsilon_{i j} C_{j}}
$$

Fig. (2). A comparison of some algorithms using influence coefficients.

The two last ternary specimens of Table 1, i.e. numbers 161 and 1189 , have a low total of $96.18 \%$ and $94.30 \%$, respectively, which means that we cannot correct for matrix effects of $3.82 \%$ and $5.70 \%$, respectively, of missing elements. Therefore, the data points of these two specimens has been omitted from Figs. (4-7) because the low totals would distort the average errors introduced by the different algorithms for the calculation of matrix effect corrections. On the other hand, Fig. (3) represents the plot of the Fe concentration (in \%) as a function of the Fe relative intensity (in \%). The data of the two low total specimens have been included in this figure to illustrate the effects of the matrix on the Fe peak intensities. In this case, the missing elements do not introduce any additional errors.

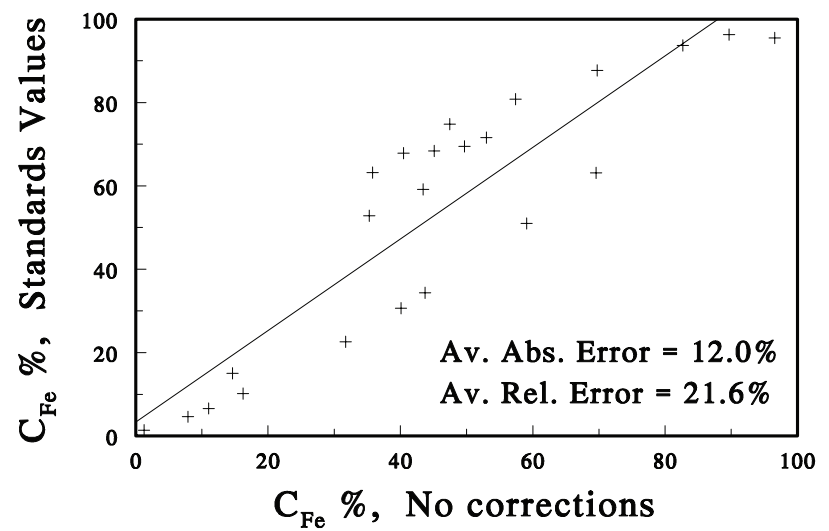

Fig. (3). Given Fe concentrations in Fe-Cr-Ni alloys plotted against concentrations calculated from uncorrected Fe intensities. Note the large absolute and relative errors.

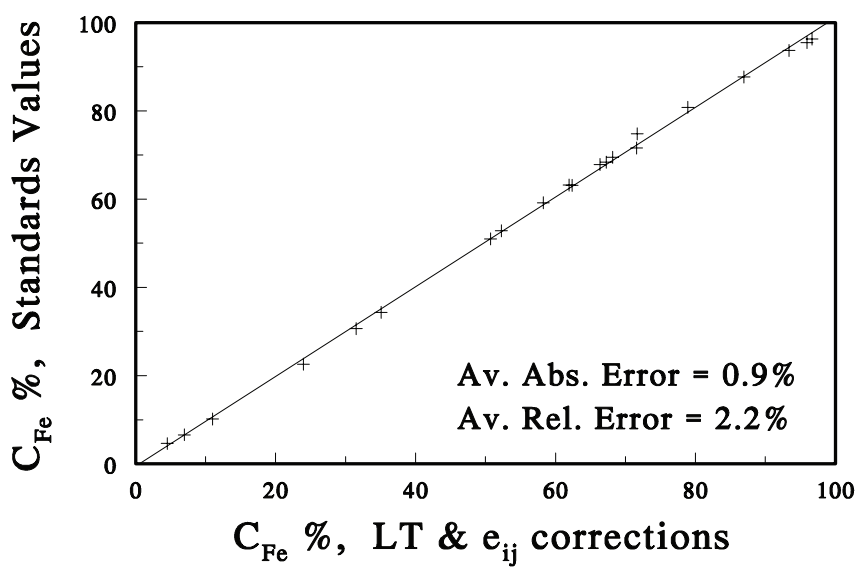

Fig. (4). Given $\mathrm{Fe}$ concentrations in $\mathrm{Fe}-\mathrm{Cr}-\mathrm{Ni}$ alloys plotted against concentrations calculated using the Lachance-Traill algorithm (LT) and empirical influence coefficients calculated by multiple regression analysis (CiREG program). Note that absolute and relative errors are still unacceptably large.

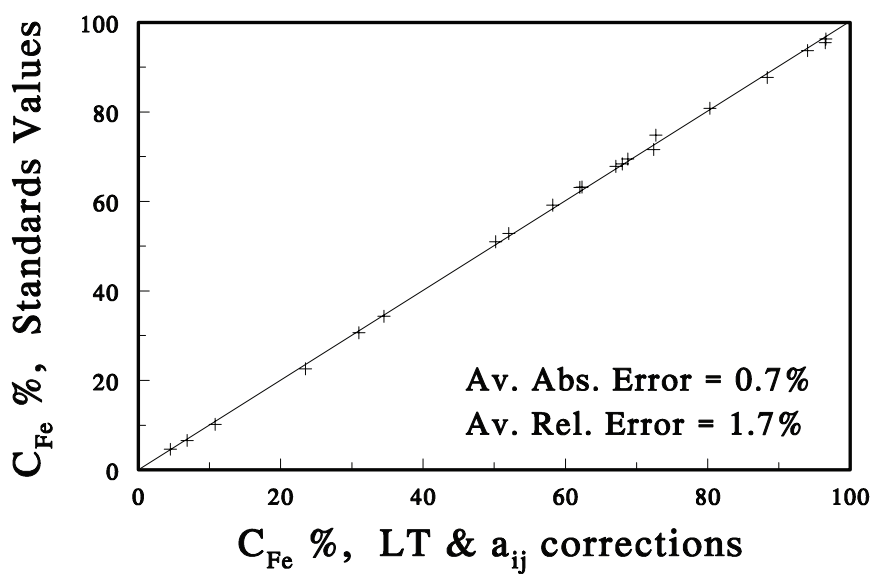

Fig. (5). Given Fe concentrations in $\mathrm{Fe}-\mathrm{Cr}-\mathrm{Ni}$ alloys plotted against concentrations calculated using the Lachance-Traill algorithm (LT) and theoretical binary influence coefficients (CiLT program). Note the very considerable improvement in absolute and relative errors.

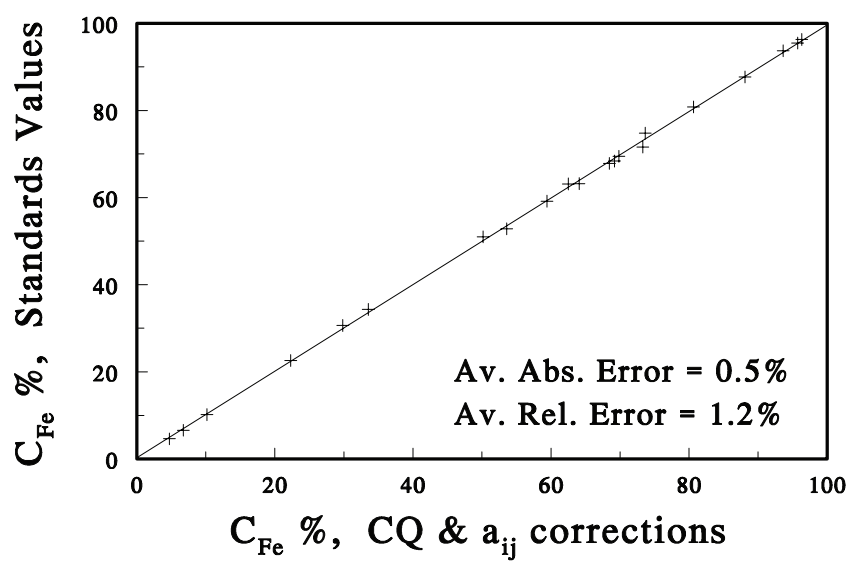

Fig. (6). Given Fe concentrations in $\mathrm{Fe}-\mathrm{Cr}-\mathrm{Ni}$ alloys plotted against concentrations calculated using the Claisse-Quintin algorithm (CQ) and theoretical binary influence coefficients (CiROU program). Note the smaller absolute and relative errors.

\section{CONCLUSION}

In this paper, it was shown that the Fundamental Algorithm firmly combines the traditional influence coefficient 


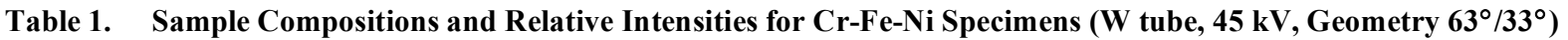

\begin{tabular}{|c|c|c|c|c|c|c|c|}
\hline \multirow{2}{*}{ Specimens } & \multicolumn{4}{|c|}{ Concentration, $\mathrm{C}$} & \multicolumn{3}{|c|}{ Relative Intensity, R } \\
\hline & $\mathrm{Cr}$ & $\mathbf{F e}$ & $\mathbf{N i}$ & Total & $\mathrm{Cr}$ & $\mathbf{F e}$ & $\mathbf{N i}$ \\
\hline \multicolumn{8}{|l|}{ Fe-Ni Binaries } \\
\hline 971 & - & 0.0462 & 0.9516 & 0.9978 & - & 0.0789 & 0.8782 \\
\hline 972 & - & 0.0659 & 0.9322 & 0.9981 & - & 0.1104 & 0.8321 \\
\hline 974 & - & 0.1018 & 0.8964 & 0.9982 & - & 0.1621 & 0.7595 \\
\hline 983 & - & 0.2263 & 0.7711 & 0.9974 & - & 0.3172 & 0.5483 \\
\hline 986 & - & 0.3067 & 0.6931 & 0.9998 & - & 0.4007 & 0.4515 \\
\hline 987 & - & 0.3431 & 0.6552 & 0.9983 & - & 0.4373 & 0.4073 \\
\hline 1159 & - & 0.5100 & 0.4820 & 0.9920 & - & 0.5907 & 0.2553 \\
\hline $126 \mathrm{~B}$ & - & 0.6315 & 0.3599 & 0.9914 & - & 0.6958 & 0.1720 \\
\hline 809B & - & 0.9549 & 0.0329 & 0.9878 & - & 0.9659 & 0.0125 \\
\hline \multicolumn{8}{|l|}{ Fe-Cr Binaries } \\
\hline 4061 & 0.0353 & 0.9627 & - & 0.9980 & 0.0617 & 0.8970 & - \\
\hline 4062 & 0.0608 & 0.9372 & - & 0.9980 & 0.1004 & 0.8270 & - \\
\hline 4065 & 0.1214 & 0.8766 & - & 0.9980 & 0.1817 & 0.6974 & - \\
\hline 4173 & 0.1900 & 0.8080 & - & 0.9980 & 0.2587 & 0.5739 & - \\
\hline 4181 & 0.2503 & 0.7477 & - & 0.9980 & 0.3326 & 0.4748 & - \\
\hline 4183 & 0.3194 & 0.6786 & - & 0.9980 & 0.4023 & 0.4048 & - \\
\hline 4184 & 0.3658 & 0.6322 & - & 0.9980 & 0.4476 & 0.3579 & - \\
\hline \multicolumn{8}{|l|}{ Fe-Ni-Cr Ternaries } \\
\hline 5074 & 0.2525 & 0.6838 & 0.0498 & 0.9861 & 0.3258 & 0.4511 & 0.0203 \\
\hline 5181 & 0.1988 & 0.6945 & 0.0996 & 0.9929 & 0.2651 & 0.4971 & 0.0416 \\
\hline 5324 & 0.2696 & 0.5280 & 0.1927 & 0.9903 & 0.3311 & 0.3529 & 0.0821 \\
\hline 5321 & 0.1988 & 0.5919 & 0.2002 & 0.9909 & 0.2582 & 0.4343 & 0.0898 \\
\hline 7271 & 0.1879 & 0.7159 & 0.0829 & 0.9867 & 0.2536 & 0.5298 & 0.0343 \\
\hline 161 & 0.1688 & 0.1501 & 0.6429 & 0.9618 & 0.2072 & 0.1460 & 0.4367 \\
\hline 1189 & 0.2030 & 0.0140 & 0.7260 & 0.9430 & 0.2263 & 0.0125 & 0.5630 \\
\hline
\end{tabular}

Data taken from: S.D. Rasberry and K.F. Heinrich, Analytical Chemistry, Vol. 46, No. 1, p. 86, January 1974

concept and the modern Fundamental-Parameters approach, thus restoring a perfect complementarity between the two approaches and giving the analyst a valuable method for mathematical matrix effect corrections. An obvious conclusion is that influence coefficients and Fundamental Parameters are not two different solutions to the Sherman equation, but two parts of the same solution.

The Fundamental Algorithm method is one of the valid solutions proposed for calculating sample compositions with the Sherman equation. This valid solution consists to calculate influence coefficients first and then concentrations by iterations, not intensities. With the FA method, the accuracy of results depends only on the quality of sample preparation and the standards used.

In addition, some algorithms making use of theoretical influence coefficients were presented. The accuracy obtainable using the different algorithms is very much dependent

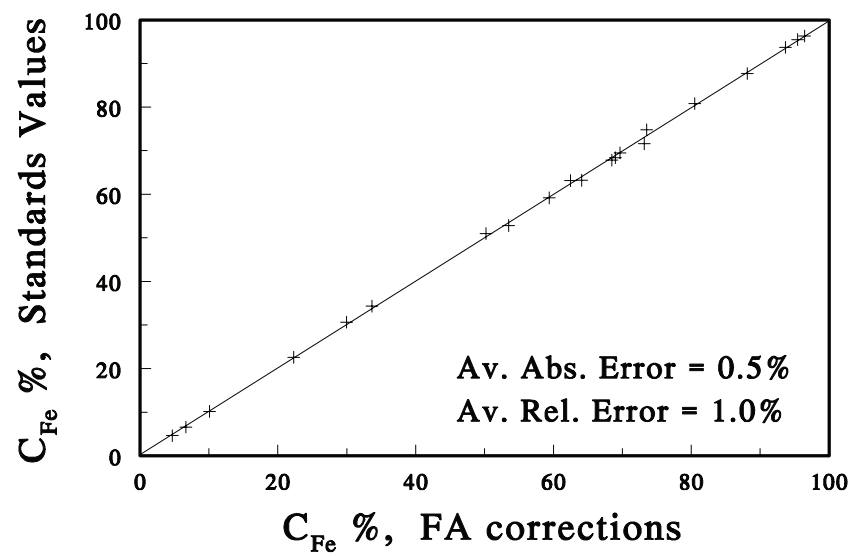

Fig. (7). Given Fe concentrations in $\mathrm{Fe}-\mathrm{Cr}-\mathrm{Ni}$ alloys plotted against concentrations calculated using the Fundamental Algorithm (FA) and theoretical multi-element influence coefficients (CiROU program). Note the very small absolute and relative errors. 
on the nature of the specimens being analyzed and concentration ranges. Examples were given and compared for the system $\mathrm{Cr}-\mathrm{Fe}-\mathrm{Ni}$ that is subject to severe absorption and enhancement effects. As a general rule it is best to make use of an algorithm that corrects for matrix effects, absorption and enhancement, and in which the magnitude of the influence coefficients can be made to vary with the concentration of the matrix elements. Influence coefficients, except in special and specific circumstances (e.g. limited concentration ranges), are not constant, and should not be treated as such if the best results are to be obtained.

\section{REFERENCES}

[1] Rousseau RM, Boivin JA. The fundamental algorithm: a natural extension of the Sherman equation, part I: theory. Rigaku J 1998; 15 (1): $13-5$

[2] Rousseau RM. Fundamental Algorithm between concentration and intensity in XRF analysis, part 2: practical application. X-Ray Spectrom 1984; 13 (3): 121-5.

[3] Rousseau RM, Willis JP, Duncan AR. Practical XRF calibration procedures for major and trace elements. X-Ray Spectrom 1996; 25: $179-11$.

[4] Rousseau RM. Concept of the influence coefficient. Rigaku J 2001; 18 (1): 8-14

[5] Sherman J. The theoretical derivation of fluorescent X-ray intensities from mixtures. Spectrochimica Acta 1955; 7: 283-24

[6] Lachance GR, Traill RJ. A practical solution to the matrix problem in X-ray analysis. Can J Spectrosc 1966; 11: 43-6.

[7] Claisse F, Quintin M. Generalization of the Lachance-Traill method for the correction of the matrix effect in X-ray fluorescence analysis. Can J Spectrose 1967; 12: 129-6.

[8] Gilfrich JV, Birks LS. Spectral distribution of X-ray tubes for quantitative X-ray fluorescence analysis. Anal Chem 1968; 40(7): $1077-4$.

[9] Criss JW, Birks LS. Calculation methods for fluorescent X-ray spectrometry, empirical coefficients vs fundamental parameters. Anal Chem 1968; 40(7): 1080-7.

[10] Rousseau R. Master thesis No. 1635, Laval University, Quebec City, Canada, 1970.

[11] De Jongh WK. X-ray fluorescence analysis applying theoretical matrix correction, stainless steel. X-Ray Spectrom 1973; 2: 151-8.

[12] Rasberry SD, Heinrich KFJ. Calibration for interelement effects in X-ray fluorescence analysis. Anal Chem 1974; 46(1): 81-9.

[13] Rousseau R, Claisse F. Theoretical alpha coefficients for the Claisse-Quintin relation for X-ray spectrochemical analysis. X-Ray Spectrom 1974; 3:31-6.

[14] Tertian R. An accurate coefficient method for X-ray fluorescence analysis. Adv X-Ray Analysis 1976; 19: 85-27.
[15] Criss JW. Fundamental-parameters calculations on a laboratory micro-computer. Adv X-Ray Analysis 1980; 23: 93-5.

[16] Rousseau RM. Fundamental Algorithm between concentration and intensity in XRF analysis, part 1: theory. X-Ray Spectrom 1984; 13 (3): 115-6.

[17] Pella PA, Feng L, Small JA. An analytical algorithm for calculation of spectral distributions of X-ray tubes for quantitative X-ray fluorescence analysis. X-Ray Spectrom 1985; 14 (3): 125-11.

[18] Shiraiwa T, Fujino N. Theoretical calculation of fluorescent X-ray intensities in fluorescent X-ray spectrochemical analysis. Jpn J Appl Phys 1966; 5(10): 886-14.

[19] Broll N, Tertian R. Quantitative X-ray fluorescence analysis by use of fundamental influence coefficients. X-Ray Spectrom 1983; 12 (1): $30-8$.

[20] Lachance GR, Claisse F. Quantitative X-Ray Fluorescence Analysis, Theory and Application. John Wiley \& Sons, Chichester, 1995.

[21] Rousseau RM. Debate on some algorithms relating concentration to intensity in XRF spectrometry. Rigaku J 2002; 19 (1): 25-10.

[22] Rousseau RM. The Fundamental Algorithm: an exhaustive study of the Claisse-Quintin algorithm and the Tertian and Lachance identities, part II: application. Rigaku J 1998; 15(2): 14-11.

[23] Les logiciels R. Rousseau inc., 28 Montmagny St., Cantley, Quebec, J8V 3J1, Canada. E-mail: xray.rousseau@videotron.ca

[24] Heinrich KFJ. The Electron Microprobe. In: McKinley TD, Heinrich KFJ, Wittry DB, Eds. Wiley: New York, 1966, pp. 296-377.

[25] Springer G, Nolan B. Mathematical expression for the evaluation of X-ray emission and critical absorption energies, and of mass absorption coefficient. Can J Spectrosc 1976; 21 (5): 134-5.

[26] Bambynek W, Crasemann B, Fink RW, et al. X-ray fluorescence yields, Auger, and Coster-Kronig transition probabilities. Rev Mod Phys 1972; 44 (4): 716-98.

[27] Hanke W, Wernisch J, Pohn C. Fluorescence yields, $\omega_{\mathrm{K}}(12 \leq \mathrm{Z} \leq$ $42)$ and ${ }^{\omega} \mathrm{L} 3(38 \leq Z \leq 79)$, from a comparison of literature and experiments (SEM). X-Ray Spectrom 1985; 14(1): 43-5.

[28] Schreiber TP, Wims AM. Relative intensity factors for K, L and M shell X-ray lines. X-Ray Spectrom 1982; 11(2): 42-4.

[29] Rousseau RM, Bouchard M. Fundamental Algorithm between concentration and intensity in XRF analysis, part 3: experimental verification. X-Ray Spectrom 1986; 15: 207-9.

[30] Lachance GR. The family of alpha coefficients in X-ray fluorescence analysis. X-Ray Spectrom 1979; 8(4): 190-6.

[31] Rousseau RM. Theory and Practice of Quantitative Analysis by $\mathrm{X}$-Ray Spectrometry, to be published within the next 3 years.

[32] Rousseau RM. Fundamental Algorithm between concentration and intensity in XRF analysis, part 2: practical application. X-Ray Spectrom 1984; 13(3): 121-5.

[33] Criss JW. Workshop W-5 on quantitative techniques and errors in XRF, 45th Annual Denver X-Ray Conference, Denver, Colorado, USA, 1996.

[34] Rousseau RM. Correction for long-term instrumental drift. X-Ray Spectrom 2002; 31(6): 401-7. 